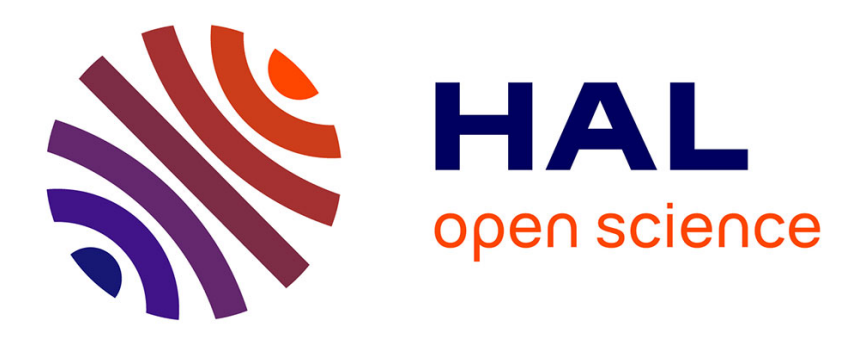

\title{
Towards Improved BCI based on Human Learning Principles
}

Fabien Lotte, Camille Jeunet

\section{To cite this version:}

Fabien Lotte, Camille Jeunet. Towards Improved BCI based on Human Learning Principles. 3rd International Winter Conference on Brain-Computer Interfaces, Jan 2015, High1 Resort, South Korea. hal-01111843

\section{HAL Id: hal-01111843 \\ https://hal.inria.fr/hal-01111843}

Submitted on 31 Jan 2015

HAL is a multi-disciplinary open access archive for the deposit and dissemination of scientific research documents, whether they are published or not. The documents may come from teaching and research institutions in France or abroad, or from public or private research centers.
L'archive ouverte pluridisciplinaire $\mathbf{H A L}$, est destinée au dépôt et à la diffusion de documents scientifiques de niveau recherche, publiés ou non, émanant des établissements d'enseignement et de recherche français ou étrangers, des laboratoires publics ou privés. 


\section{Towards Improved BCI based on Human Learning Principles}

\author{
Fabien LOTTE \\ Inria - LaBRI, France \\ Email: fabien.lotte@inria.fr
}

\author{
Camille JEUNET \\ University of Bordeaux - Inria, France \\ Email: camille.jeunet@inria.fr
}

\begin{abstract}
Although EEG-based BCI are very promising for numerous applications, they mostly remain prototypes not used outside laboratories, due to their low reliability. Poor BCI performances are partly due to imperfect EEG signal processing algorithms but also to the user, who may not be able to produce reliable EEG patterns. This paper presents some of our current work that aims at addressing the latter, i.e., at guiding users to learn BCI control mastery. First, this paper identifies some theoretical (based on human learning psychology models) and practical limitations of current standard BCI training approaches and thus the need for alternative ones. To try to address these limitations, we conducted a study to explore what kind of users can use a BCI and why, and will present the main results. We also present new feedback types we designed to help users to learn BCI control skills more efficiently.
\end{abstract}

\section{INTRODUCTION}

ElectroEncephaloGraphy (EEG)-based Brain-Computer Interfaces (BCI) make computer control possible without any physical activity [1]. As such, they have promised to revolutionize many application areas, including assistive devices or human-computer interaction [1]. Despite this promising potential, such revolutions have not been delivered yet, and BCI are still barely used outside laboratories [1]. This is mainly due to the substantial lack of reliability of current BCI [1]. In particular, BCI often inaccurately recognize the users' mental commands [2] whereas roughly $20 \%$ of BCI users cannot control the system at all (the so-called BCI illiteracy/deficiency) [3].

To operate a BCI, the user has to produce EEG patterns, typically using mental imagery tasks ${ }^{1}$, which the machine has to recognize by using signal processing. So far, to address the reliability issue of BCI, most research efforts have been focused on EEG signal processing only [3]. Thus, the reliability issue of BCI is unlikely to be solved by focusing on signal processing alone. Indeed, BCI control is known to be a skill that needs to be learned and mastered by the user [1]. This means that 1) the BCI performances of a user become better with practice and thus that 2) the user needs to learn how to produce stable, clear and distinct brain activity patterns to successfully control a BCI. With poor user BCI control skills, even the best signal processing algorithms will fail to recognize the user's mental commands. Unfortunately, how to train users to $\mathrm{BCI}$ control has been rather scarcely studied so far. Thus, the best way to train users to master BCI control skills is still unknown [1][3].

\footnotetext{
${ }^{1}$ Note that BCI based on Event Related Potentials are not considered in this paper as they involve very little or no human training [1]
}

This paper aims at convincing the reader that changing $\mathrm{BCI}$ design to enable their users to master BCI control skills is a very promising direction to improve BCI reliability. Indeed, this paper first identifies the theoretical and practical limitations of current standard BCI training protocols, which may explain, at least in part, the current high rate of BCI illiteracy/deficiency and their overall modest performance. It then presents our ongoing work towards improving these training protocols. It notably presents some results about what kind of users can use mental imagery-based BCI and why. It also introduces new feedback types and new training environments targeted at improving the user's understanding of BCI use as well as his/her motivation to learn the BCI skill.

\section{THEORETICAL AND PRACTICAL LIMITATIONS OF CURRENT BCI TRAINING APPROACHES}

BCI control being a skill, it has to be mastered by the BCI user [4]. Typically, standard BCI training is performed by asking the user to control an object on screen by modulating his/her brain activity in a specific way (e.g., using Motor Imagery (MI)). The feedback provided to the user about his/her task performance is thus generally a uni-modal (generally visual) feedback indicating the mental task recognized by the classifier together with the confidence in this recognition and is represented by an extending bar or a moving cursor [4]. The user is generally trained following a synchronous protocol, i.e., the user is required to do specific tasks (e.g., left hand MI) in specific time periods only. The same protocol is usually repeated until the user has learned the BCI skill, i.e., until he/she has achieved a given classification accuracy.

\section{A. Theoretical limitations}

Unfortunately, such standard training approaches satisfy very few of the guidelines provided by human learning and instructional design principles to ensure an efficient learning of a skill [5]. For instance, a typical BCI training session provides only corrective feedback (indicating whether the learner performed the task correctly), using fixed and somewhat boring training tasks identically repeated until the user has learned the BCI skill, with these training tasks being provided in a synchronous way. In contrast, human learning and instructional design principles recommend to provide an explanatory feedback (indicating what was right or wrong about the task performed by the user) that is goal-oriented (i.e., indicating a gap between the current performance and the desired level of performance). The feedback could also benefit from multimodality and an engaging and challenging 
environment with adaptive difficulty [5]. In short, current standard BCI training approaches are theoretically suboptimal, and are unlikely to enable efficient learning of the BCI skill.

Moreover, according to Keller [6], it is necessary to consider the user's motivational and cognitive state to ensure he/she can learn and perform efficiently, irrespectively of the task. Indeed, according to Keller's theory, optimizing motivational factors - Attention (triggering a person's curiosity), Relevance (the compliance with a person's motives or values), Confidence (the expectancy for success), and Satisfaction (by intrinsic and extrinsic rewards) - leads to more user efforts and thereby a better performance. Additionally, considering the cognitive factors - the limited user's working memory capacity (requiring to minimize the amount of skill-unrelated information), the way information is actively processed by $\mathrm{him} / \mathrm{her}$ (requiring to make relevant information salient) and the existing knowledge in his/her long-term memory (requiring to relate the to-be-learned skill to existing knowledge) - leads to a more efficient skill acquisition. Again, these different factors are typically not considered in BCI training protocols, or only very few of them, leading to theoretically suboptimal training protocols [7].

\section{B. Practical limitations}

The limitations mentioned above are only theoretical limitations though and one may wonder whether they translate into actual practical limitations. Indeed, those standard training protocols still enabled many users to gain control of a BCI system. It would therefore be interesting to study what the impact of current BCI training protocols on BCI performance and illiteracy/deficiency is. Unfortunately, there are many reasons why a given user may not gain BCI control: poor EEG signal-to-noise ratio, non-stationarity of the signals or nonaccess to the relevant brain signals due to the way the cortical neurons of the user are oriented, among many others. As such, a failure to use the BCI may have many possible causes related to EEG signals but not to the training protocol. Therefore, to work around these issues, we proposed to study standard BCI training protocols without EEG signals, i.e., without a BCI [8]. In particular, we studied how people could learn to do two simple motor tasks using the same training tasks and feedback as those given to MI BCI users (see Figure 1). More precisely, we asked subjects to learn to draw on a graphic tablet a triangle and a circle (the correct size, angles and speed of drawing of these two shapes being unknown to the subject) that can be recognized by the system, using a synchronous training protocol and an extending bar as feedback, like for MI-based BCI training. Our results show that most subjects (out of $\mathrm{N}=20$ subjects) improved with this feedback and practice (i.e., the shapes they draw are increasingly more accurately recognized by the system), but that $15 \%$ of them completely fail to learn how to draw the correct shapes, despite the simplicity of the motor tasks. This suggests that part of BCI illiteracy/deficiency is likely due to the training protocols currently used.

\section{Alternative approaches}

Fortunately, some groups have explored alternative BCI training protocols, which satisfy some of the guidelines about efficient human learning [5]. For instance, [9] showed that a

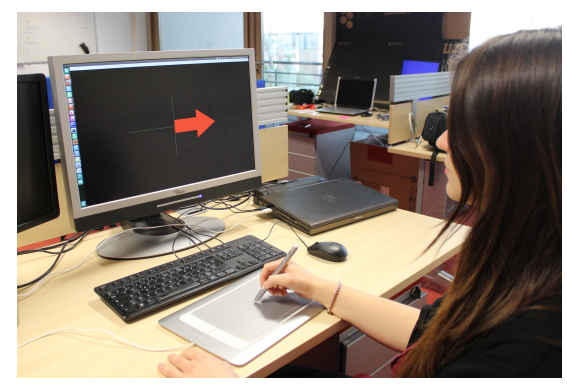

Fig. 1. A user learning to perform simple motor tasks, using a pen to draw on a graphic tablet, with the same feedback as used for MI BCI training.

richer feedback, based on a 2D topography of cortical activation could increase MI-based BCI performances. Using BCI with game-like feedback environments also led to increased BCI performances [10]. Some studies showed that biased feedback (i.e., making the user believe he/she did better than what he/she actually did) or positive feedback only (i.e., only providing feedback when the task was performed correctly) can improve performances, at least for new or inexperienced BCI users [11][12]. Finally, some groups have also successfully explored progressive BCI training tasks [13][14]. These works further confirm that improving BCI training protocols can improve BCI performance. Unfortunately, such alternative training protocols are typically not used by the BCI community and still satisfy only a small subset of the guidelines from human learning, and many recommendations are yet to be explored. In the following sections we present our ongoing work to design BCI training protocols satisfying such guidelines.

\section{UNDERSTANDING WHO CAN USE CURRENT BCI AND WHY}

BCI deficiency and more generally the huge variability in users' ability to control a MI-BCI led to some studies looking for psychological [15] and neurophysiological [2] predictors of MI-BCI performance. However, most of them are based on one-session (i.e., one day) experiments only, while several sessions are required to learn to master a BCI. Moreover, these studies only consider MI while other mental imagery tasks have been shown to be more efficient [16]. Thus, in order to address these limitations, we looked for predictors based on data collected over 6 sessions (i.e., over 6 days) during which participants had to learn to perform 3 mental imagery tasks: mental rotation, left-hand motor imagery and mental subtraction [16]. Our results $(\mathrm{N}=18)$ show that 1) performances are strongly correlated with users' spatial abilities and 2) we can reliably predict these performances using a model including different psychological factors (like abstractedness, self-reliance or tension). These results are very encouraging as they could lead to reflexions about 1) exercises to improve users' spatial abilities and 2) solutions to take into account users' cognitive and personality profiles in BCI training approaches [17].

\section{NEW FEEDBACK AND EEG VISUALIZATION TOOLS}

As mentioned above, to ensure an efficient learning, the provided feedback should be explanatory, engaging and take benefit from multimodality [5]. This section reports on the design and evaluation of such kinds of BCI feedback. 


\section{A. Multi-user feedback}

Making a learning task more engaging and motivating can be achieved by challenging the learner [5], and setting the learning task in a social context [18]. We therefore explored MI-based BCI training in a multi-user context, with multi-user feedback [19]. We proposed a BCI game in which users had to push a ball towards a targeted goal located on the left or right of the screen, by imagining left or right hand movements respectively. Users could play the game with another BCI user, the ball moving according to the sum of the BCI outputs from the two users. Users were provided with their own feedback (the classifier output for their EEG signals) as well as the combined output, i.e., the sum of classifier outputs from both users. The game could be in a collaborative version, the two users having to push the ball in the same direction, or in a competitive version, the two users having to push the ball in opposite directions (see Figure 2). In an evaluation study in which we compared a single player version of the game, to the multiplayer collaborative version of it, we observed that the multiplayer version could improve BCI performances for a specific category of users. In particular, for each pair of users, the user who was the best at BCI had significantly increased classification performances as compared to the single player version of the game, while the classification accuracy for the other user did not change. This suggests that such a multiuser feedback and environment could increase BCI reliability for some users.

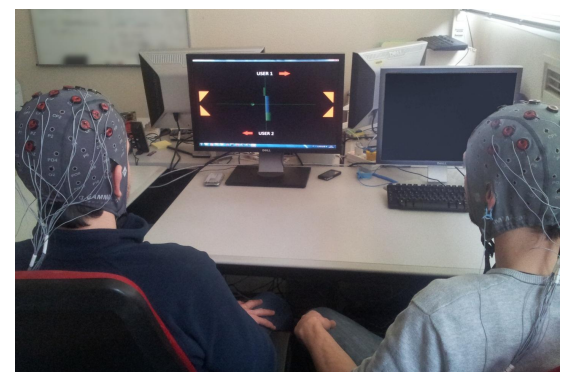

Fig. 2. Two users competing in a Motor Imagery-based BCI game.

\section{B. Multimodality}

As mentioned above, most training protocols involve visual feedback. Yet, both theoretical [5] and practical [20] evidences argue for the use of other sensori-modalities that could be more adapted to BCI-based applications. Among these modalities, the tactile channel seems to be a good candidate as it is often not overtaxed in interaction contexts, contrary to the visual and auditory ones. Furthermore, increasing motivation, for instance by creating more appealing training environments, has also been shown to be efficient to increase users' performances [5]. However, the combination of these two parameters (tactile feedback and appealing environments) had never been tested in a BCI training protocol. Thus, our study [21] aimed at comparing a standard visual feedback with an equivalent tactile feedback in an appealing training environment containing visual distractors (to mimic an interaction context in which the visual channel is overtaxed). Users had to learn to perform motor-imagery tasks as well as a counting task, and received either a visual or vibrotactile feedback (see Figure 3). Our main result $(\mathrm{N}=18)$ is the fact that people receiving tactile feedback perform significantly better (in MI and counting task). Such results should encourage the BCI community to replace standard BCI protocols by more motivating training environment and multimodal feedback, as visual feedback may not be adapted to visual interaction applications.

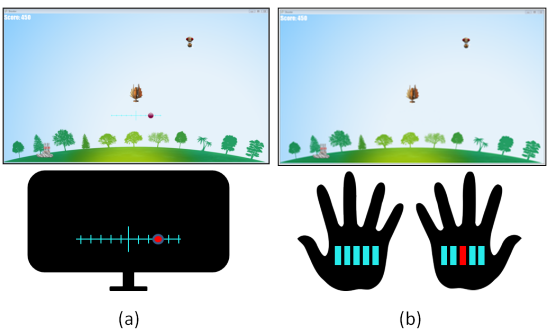

Fig. 3. Illustration of the combination of an appealing training environment and a vibrotactile feedback provided on the palm of the hand using an array of vibrators (right, compared to a standard visual feedback, left)

\section{Explanatory feedback}

Another desired property of a good training feedback, is to be explanatory. In other words, the feedback should provide enough information so that the user can understand what mental imagery strategies lead to correctly recognized mental commands, and why. With this objective in mind, we designed two new real-time brain activity visualization tools, the Mind-Mirror and Teegi (see Figure 4), that can be used as explanatory BCI feedback.
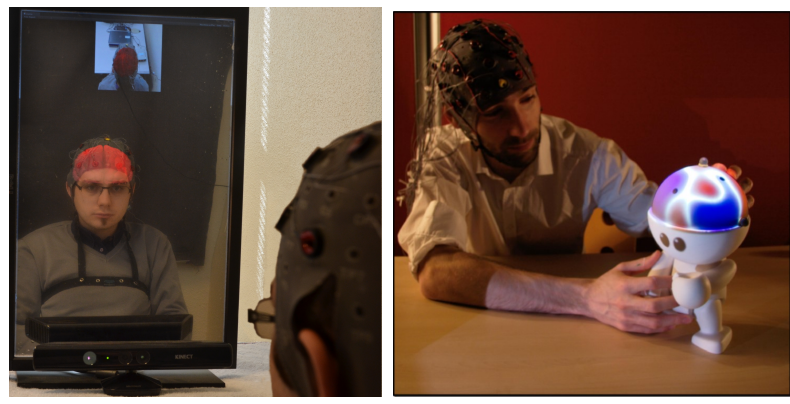

Fig. 4. Left: the Mind Mirror, Right: Teegi, two new real-time visualization tools of the user's own brain activity, based on augmented reality.

1) Mind-Mirror: The Mind-Mirror enables its users to visualize their own brain activity in real-time, in their own head [22]. It uses augmented reality and head-tracking to overlay a representation of an active brain on top of the user's head, seen in a semi-reflective screen (see Figure 4, left). This gives the illusion that the user can see his/her own brain in his head, in a mirror, in activity, the EEG power from different channels being represented in real-time on the surface of the brain. The Mind-Mirror was tested as a feedback to train users to control an attention-based BCI in which the user had to perform concentration or relaxation tasks to control two different commands. The Mind-Mirror was compared to a classical gauge feedback for the same task. Results showed that users found the Mind-Mirror to be indeed an engaging and innovative visualization tool. In terms of performance however, it was not better than the classical gauge feedback. This suggests that selecting and visually enhancing the relevant information is probably necessary to ease the user perception and understanding of his/her own brain activity. 
2) Teegi: Teegi is a tangible EEG interface, designed to enable users that are naive with EEG to get to know more about how EEG works, in an accessible and engaging way [23]. As with the Mind-Mirror, Teegi enables users to visualize their own brain activity in real-time (See Figure 4, right). Contrary to the former, Teegi is based on educational design principles to ease the user understanding of brain activity. In particular, it is based on a tangible system, the user's brain activity being projected on the head of a puppet (with a friendly and humanoid appearance), that can be easily manipulated, to favor exploration. EEG signals can also be filtered to reveal some specific EEG phenomenons (e.g., sensorimotor rhythms), hence enhancing the relevant EEG signals. Teegi was so far explored only for scientific outreach and education, to help users to know more about the brain and EEG - with success. In the future, we plan to use it as an explanatory BCI feedback.

\section{CONCLUSION}

In this paper we argued that, in order to bring BCI reliability to the next level, it is necessary to study further how users can learn to gain BCI control. We notably showed that current standard BCI training approaches have many limitations, both theoretical, since they do not satisfy guidelines from human learning theories, and practical, since they may fail to teach people simple motor tasks. To better identify how to improve BCI training protocols, we tried to design a model relating the personality and cognitive profile of the user to his/her mental imagery BCI performances, obtained with standard protocols. We notably showed that BCI performances are correlated to the user's abstractedness, self-reliance, tension and ability in mental rotation, thus providing us with specific insights about what to improve and how. To ensure that BCI training protocols are more in line with guidelines from human learning theories, we also proposed new feedback types. We notably showed that both multi-user feedback and multimodality (exploiting vibrotactile feedback) can improve BCI performances. We are also exploring new ways to display EEG activity to BCI users, to help them understand their EEG patterns, for which we proposed the Mind-Mirror and Teegi augmented reality-based visualization tools.

In the longer term, it would be necessary to build a comprehensive training framework to teach anyone to gain $\mathrm{BCI}$ control. An interesting approach to do so would be to design an Intelligent Tutoring System (ITS) [24]. Indeed, such systems would allow to determine each user's profile, and then propose a training protocol adapted to this profile. Moreover, it could be improved by adapting in real time the training protocol to the emotional, motivational and cognitive states of the user in order to optimize the learning phase (i.e. make it more pleasant, more efficient and faster). We hope the BCI community will join these research efforts, in order to reach much more reliable BCI systems, and thus broaden the application areas for these technologies.

\section{REFERENCES}

[1] J. Wolpaw and E. Wolpaw, Brain-computer interfaces: principles and practice. Oxford University Press, 2012.

[2] B. Blankertz, C. Sannelli, S. Halder, E. Hammer, A. Kübler, K.-R Müller, G. Curio, and T. Dickhaus, "Neurophysiological predictor of SMR-based BCI performance," NeuroImage, vol. 51, no. 4, 2010.
[3] B. Allison and C. Neuper, "Could anyone use a BCI?" in BrainComputer Interfaces, ser. Human-Computer Interaction Series, D. S. Tan and A. Nijholt, Eds. Springer London, 2010, vol. 0, pp. 35-54.

[4] C. Neuper and G. Pfurtscheller, Brain-Computer Interfaces. The Frontiers Collection, 2010, ch. Neurofeedback Training for BCI Control

[5] F. Lotte, F. Larrue, and C. Mühl, "Flaws in current human training protocols for spontaneous brain-computer interfaces: lessons learned from instructional design," Frontiers in Human Neuroscience, vol. 7, no. $568,2013$.

[6] J. Keller, "An integrative theory of motivation, volition, and performance," Technology, Instruction, Cognition, and Learning, vol. 6, no. 2, pp. 79-104, 2008.

[7] C. Mühl, R. Scherer, A. Lécuyer, L. Perronnet, M. Grosse-Wentrup, and F. Lotte, "Which factors drive successful BCI skill learning?" in 6th International BCI conference, 2014.

[8] C. Jeunet, A. Cellard, S. Subramanian, M. Hachet, B. N'Kaoua, F. Lotte et al., "How well can we learn with standard bci training approaches? a pilot study." in 6th International BCI Conference, 2014.

[9] H.-J. Hwang, K. Kwon, and C.-H. Im, "Neurofeedback-based motor imagery training for brain-computer interface (BCI)," Journal of Neuroscience Methods, vol. 179, no. 1, pp. 150 - 156, 2009.

[10] F. Lotte, J. Faller, C. Guger, Y. Renard, G. Pfurtscheller, A. Lécuyer, and R. Leeb, "Combining BCI with Virtual Reality: Towards new applications and improved BCI," in Towards Practical Brain-Computer Interfaces. Springer Berlin Heidelberg, 2013, pp. 197-220.

[11] A. Kübler, N. Neumann, J. Kaiser, B. Kotchoubey, T. Hinterberger, and N. Birbaumer, "Braincomputer communication: self-regulation of slow cortical potentials for verbal communication," Arch Phys Med Rehabil, vol. 82, p. 15331539, 2001.

[12] A. Barbero-Jimenez and M. Grosse-Wentrup, "Biased feedback in braincomputer interfaces," Journal of NeuroEngineering and Rehabilitation 7(34), vol. 7, no. 34, pp. 1-4, 2010.

[13] D. McFarland, W. Sarnacki, and J. Wolpaw, "Electroencephalographic (EEG) control of three-dimensional movement," Journal of Neural Engineering, vol. 7, no. 3, 2010.

[14] C. Vidaurre and B. Blankertz, "Towards a cure for BCI illiteracy," Brain Topography, vol. 23, pp. 194-198, 2010.

[15] E. M. Hammer, S. Halder, B. Blankertz, C. Sannelli, T. Dickhaus, S. Kleih, K.-R. Müller, and A. Kübler, "Psychological predictors of smr-bci performance," Biological psychology, vol. 89, pp. 80-86, 2012.

[16] E. V. Friedrich, C. Neuper, and R. Scherer, "Whatever works: A systematic user-centered training protocol to optimize brain-computer interfacing individually." PloS one, vol. 8(9), p. e76214, 2013.

[17] C. Jeunet, F. Lotte, M. Hachet, S. Subramanian, and B. N'Kaoua, "Determining personality and cognitive profiles to predict mental imagerybased bci performance," in preparation.

[18] R. B. Zajonc et al., Social facilitation. Research Center for Group Dynamics, Institute for Social Research, University of Michigan, 1965.

[19] L. Bonnet, F. Lotte, and A. Lécuyer, "Two brains, one game: Design and evaluation of a multi-user BCI video game based on motor imagery," IEEE Transactions on Computational Intelligence and AI in Games, vol. 5, no. 2, pp. 185-198, 2013.

[20] R. Leeb, K. Gwak, D.-S. Kim, J. d. Millan et al., "Freeing the visual channel by exploiting vibrotactile bci feedback," in Engineering in Medicine and Biology Society (EMBC), 2013 35th Annual International Conference of the IEEE. IEEE, 2013, pp. 3093-3096.

[21] C. Jeunet, C. Vi, D. Spelmezan, B. N'Kaoua, F. Lotte, and S. Subramanian, "Tactile feedback for motor-imagery based bci," in submitted.

[22] J. Mercier-Ganady, F. Lotte, E. Loup-Escande, M. Marchal, and A. Lecuyer, "The mind-mirror: See your brain in action in your head using eeg and augmented reality," in Virtual Reality (VR), 2014 iEEE. IEEE, 2014, pp. 33-38.

[23] J. Frey, R. Gervais, S. Fleck, F. Lotte, and M. Hachet, "Teegi: tangible eeg interface," in Proceedings of the 27th annual ACM symposium on User interface software and technology. ACM, 2014, pp. 301-308.

[24] R. Nkambou, J. Bourdeau, and R. Mizoguchi, Advances in intelligent tutoring systems. Springer, 2010, vol. 308. 